\title{
Travelling Wave Solutions of a System of Nonlinear Diffusion Equations with Integral Term
}

\author{
P. KNABNER and L.v. WOLFERSDORF
}

The existence of travelling wave solutions with compact support is shown for a system of nonlinear non-local degenerate diffusion equations of population dynamics. Besides the behaviour of an important class of particular solutions of this type is investigated analytically and numerically.

Key words: Nonlinear diffusion equations, travelling waves

AMS subject classification: $35 \mathrm{Q} 20,45 \mathrm{KOS}$

\section{Introduction}

Recently, in population dynamics Mimura and YAMAGUTI [11], NAGAI [12], and NAGAI and MIMURA [13] proposed and studied the Cauchy problem for the nonlinear degenerate diffusion equation with integral term

$$
u_{t}=\left(u^{m}\right)_{x \cdot x}-\left[\left\{\int_{-\infty}^{+\infty} K(x-y) u(y, t) d y\right\} u\right]_{x}, m>1,
$$

where $K(x)$ is a piecewise continuous kernel with a finite jump at $x=0$. According to GURNEY and NISBET [3] and GURTIN and MAC CAMY [4] the first term on the right - hand side of (1) describes a local dispersion of a population whereas the second term has been introduced by MIMUR^ and YAMAGUTI [11] for describing a non-local aggregation effect of the population. The balance of both effects makes the existence of travelling wave solutions with compact support for equation (1) possible. So IKEDA [5] and MIMURA and SATSUMA [10] constructed explicit equilibrium and travelling wave solutions with compact support for particular integral terms in diffusion equations of the form (1), especially for the case $m=2$. Further in NAGAI and MIMURA $[14,15]$ the asymptotic behaviour of the interfaces of solutions with compact support and of the solutions themselves is investigated. Besides, IKEDA and NAGAI [6] considered the stability of localized stationary solutions of equations of this type. Stability properties of more general degenerate diffusion equations with drift functionals modelling aggregation are dealt with by ALT [1] and GREENBERG and ALT [2].

In the present paper the existence of travelling wave solutions with compact support is studied for a related system of diffusion equations to (1) with general sufficiently smooth integral terms. Corresponding results for the equation (1) are obtained in the recent paper [16] by one of the authors. Here these results are extended to general systems with piecewise constant kernels and to special classes of such systems with general 
piecewise continuously differentiable kernels, respectively. Moreover, the particular travelling wave solutions of the equation (1) with piecewise constant kernels given in [16] are investigated analytically and numerically. Especially we study the behaviour of these solutions as the exponent $m$ in equation (1) tends to the exceptional value $m=2$ of the theory and show numerical results for the convergence of these solutions if $m$ goes to 2 in a suitable way.

The paper is based on the report [8] where also simple examples and extensions to a special more-dimensional equation and another type of system of diffusion equations in the form (1) can be found. The report was written during the second author's stay at the University of Augsburg, Institute of Mathematics, under a contract with the Deutsche Forschungsgemeinschaft in December 1988. This author would like to thank Prof Dr. K.H. Hoffmann from the University of Augsburg for the invitation to this visit and the hospitality during it.

\section{Statement of problem}

We deal with the system of equations

$$
\frac{\partial u_{i}}{\partial t}=k_{i} \frac{\partial^{2}}{\partial x^{2}}\left(u_{i}^{m j}\right)-\left[\left\{\sum_{j=1}^{n} \int_{-\infty}^{+\infty} K_{i j}\left(x-y-\gamma_{i j} t\right) u_{j}(y, t) d y\right\} u_{i}\right] \times
$$

for functions $u_{j}(x, t)(x \in \mathbb{R}, t>0 ; j=1, \ldots, n)$, where $k_{j}>0, m_{i}>1$ are given parameters and $\gamma_{i j} \in \mathbb{R}$ are unknown parameters to be chosen in such a way that travelling wave solutions of the form

$$
u_{i}=\varphi_{i}\left(x-c_{i} t\right), i=1, \ldots, n
$$

with a common compact support interval $[0, a]$ exist, i.e. $\varphi_{j}(s)=0$ on $(-\infty, 0) \cup(a,+\infty)$ and $\varphi_{i}(s) \geq 0$ with " $>$ " instead of " 2 " for at least one index $i$ on $(0, a)$. We suppose that the functions $\varphi_{i}$ together with the derivatives $\left(\varphi_{i}^{m_{i}}\right)^{\prime}$ are continuous on $\mathbb{R}$ and the $\varphi_{i}$ are twice continuously differentiable on $(0, a)$. The kernels $K_{i j}$ have the form

$$
K_{i j}(s)=\left\{\begin{array}{l}
K_{i j}^{(1)}(s) \text { as } s<0 \\
K_{i j}^{(2)}(s) \text { as } s>0,
\end{array}\right.
$$

where $K_{i j}^{(1)}, K_{i j}^{(2)}$ are continuously differentiable functions on $(-\infty, 0]$ and $[0,+\infty)$, respec tively, having non-positive derivatives $\left(K_{i j}^{(1)}\right)^{\circ},\left(K_{i j}^{(2)}\right)^{\prime} \leq 0$ there and non-negative jumps at $s=0$

$$
\Delta_{i j}=\lim _{s \rightarrow-0} K_{i j}^{(1)}(s)-\lim _{s \rightarrow+0} K_{i j}^{(2)}(s) \geq 0 \quad(i, j=1, \ldots, n) .
$$

Inserting the ansatz (3) in (2), we see that there should be $\gamma_{i j}=c_{i}-c_{j}$ and after integrating, we obtain the integro-differential equations for $\varphi_{i}$ in $(0, a)$

$$
\kappa_{i} m_{i} \varphi_{i}^{m_{i}-2}(s) \varphi_{j}^{\prime}(s)-\sum_{j=1}^{n} \int_{0}^{a} K_{i j}(s-\sigma) \varphi_{j}(\sigma) d \sigma+c_{i}=0
$$

with the boundary conditions $\varphi_{i}(0)=\varphi_{j}(a)=0$. Introducing further the new unknown func- 
tions $\Phi_{i}=\varphi_{i}^{m_{i}-1}$ and the known parameters $p_{j}=1 /\left(m_{i}-1\right), \lambda_{i}=k_{i} m_{i} /\left(m_{i}-1\right)=k_{i}\left(1+p_{i}\right)$, the system (6) goes over into the system for $\Phi_{i}$

$$
\lambda_{j} \Phi_{j}(s)-\sum_{j=1}^{n} \int_{0}^{a} K_{i j}(s-\sigma) \Phi_{j}^{p_{j}}(\sigma) d \sigma+c_{i}=0
$$

with the boundary conditions $\Phi_{j}(0)=0=\Phi_{i}(a)$ again. This in turn is equivalent to the two-point boundary value problem

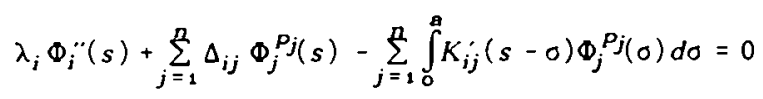

in $(0, a)$ with $\Phi_{j}(0)=0=\Phi_{j}(a)$ and the relation for the speeds $c_{i}$

$$
c_{i}=\sum_{j=1}^{n} \int_{0}^{a} K_{i j}^{(1)}(-\sigma) \Phi_{j}^{p_{j}}(\sigma) d \sigma-\lambda_{i} \Phi_{j}(0)
$$

The boundary value problem (7) can be reduced to the integral equation system

$$
\Phi_{i}(s)=\sum_{j=1}^{n} \int_{0}^{a} G_{i j}(s, \sigma) \Phi_{j}^{p_{j}(\sigma) d o, 0 \leq s \leq a,}
$$

with the kernels

$$
G_{i j}(s, \sigma)=\left(\Delta_{i j} / \lambda_{j}\right) G_{0}(s, \sigma)-\lambda_{i}^{-1} \int_{0}^{a} G_{0}(s, \rho) K_{i j}^{\prime}(\rho-\sigma) d \rho
$$

where

$$
G_{0}(s, \sigma)=\left\{\begin{array}{l}
s(a-\sigma) / a \text { as } 0 \leq s \leq \sigma \leq a \\
(a-s) \sigma / a \text { as } 0 \leq 0 \leq s \leq a
\end{array}\right.
$$

is the well-known Green's function.

In the sequel we substitute $s=a t, \sigma=a t$ and $\Psi_{i}(t)=\Phi_{i}(a t)$ in equation (8) and finally obtain the integral equation system

$$
\Psi_{i}(t)=\sum_{j=1}^{n} \int_{0}^{1} k_{i j}(t, \tau ; a) \Psi_{j}^{P j}(\tau) d \tau, 0 \leq t \leq 1,
$$

with the kernels $k_{i j}(t, \tau ; a)=a G_{i j}(a t, a \tau)$, i.e.

$$
k_{i j}(t, \tau ; a)=k_{i j}^{(o)}(t, \tau ; a)+k_{i j}^{(1)}(t, \tau ; a),
$$

where

$$
k_{i j}^{(o)}(t, \tau ; a)=\frac{a^{2} \Delta_{i j}}{\lambda_{i}} g_{0}(t, \tau) \text { and } k_{i j}^{(1)}(t, \tau ; a)=-\frac{a^{3}}{\lambda_{i}} \int_{0}^{1} g_{0}(t, \rho) K_{i j}(a(p-\tau)) d \rho,
$$

with the normalized Green's function

$$
g_{0}(t, \tau)=\left\{\begin{array}{l}
t(1-\tau) \text { as } 0 \leq t \leq \tau \leq 1 \\
\tau(1-t) \text { as } 0 \leq \tau \leq t \leq 1
\end{array}\right.
$$

The problem of existence of travelling wave solutions of the form (3) for the differential equations (2) is now equivalent to seek non-trivial non-negative solution systems of the integral equations $(9)$ in some finite interval $[0, a]$. 


\section{General case}

In the general case

$$
m_{i} \begin{cases}>2 & \text { for } i=1, \ldots, k \\ =2 & \text { for } i=k+1, \ldots, I \\ <2 & \text { for } i=1+1, \ldots, n\end{cases}
$$

we restrict ourselves to piecewise constant kernels

$$
K_{i j}(s)= \begin{cases}\alpha_{i j} \text { as } s<0 \\ \beta_{i j} \text { as } s>0\end{cases}
$$

with $\Delta_{i j}=\alpha_{i j}-\beta_{i j} \geq 0 \quad(i, j=1, \ldots, n)$. Then the two-point boundary value problem (7) is a pure differential equation problem and the integral equation system (9) has the form of an eigenvalue problem

$$
\Psi=v A_{0} \Psi
$$

for the vector function $\Psi=\left(\Psi_{1}, \ldots, \Psi_{n}\right)$ in $[0,1]$ with the eigenparameter $\nu=a^{2}>0$ and the operator $A_{\mathrm{o}}$ defined by

$$
\left(A_{0} z\right)(t)=\left(\sum_{j=1}^{n}\left(\Delta_{i j} / \lambda_{i}\right) \int_{0}^{1} g_{0}(t, \tau) z_{j}^{p j}(\tau) d \tau\right)(i=1, \ldots, n)
$$

for $z=\left(z_{1}, \ldots, z_{n}\right)$, where

$$
\begin{array}{ll}
p_{i} \in(0,1) & \text { if } i=1, \ldots, k \\
p_{j}=1 & \text { if } i=k+1, \ldots, l \\
p_{i}>1 & \text { if } i=1+1, \ldots, n
\end{array} \quad(0 \leq k \leq 1 \leq n) .
$$

The operator $A_{0}$ is completely continuous in the Banach space $C^{n}[0,1]$ of continuous $n$-dimensional vector functions, leaves the cone $K^{n}[0,1]$ of non-negative vector functions from $C^{n}[0,1]$ invariant, maps the zero element into itself and possesses the linear minorants $B_{0}$ defined by

$$
\left(B_{\mathrm{O}} z\right)(t)=\left\{\begin{array}{l}
\left(\sum_{j=1}^{l}\left(\Delta_{i j} / \lambda_{j}\right) \int_{0}^{1} g_{\mathrm{o}}(t, \tau) z_{j}(\tau) d \tau\right)_{j=1, \ldots, 1} \\
(0)_{i=1+1, \ldots, n}
\end{array}\right.
$$

in the intersection of the cone $K^{n}[0,1]$ with the unit ball of $C^{n}[0,1]$ and

$$
\left(B_{\mathrm{o}} z\right)(t)=\left\{\begin{array}{l}
\left.(0)_{i=1, \ldots, k},{ }^{1}\left(\Delta_{i j} / \lambda_{i}\right) \int_{0}^{1} g_{0}(t, \tau) z_{j}(\tau) d \tau\right)_{i=k+1, \ldots, 1} \\
\left(\sum_{j=k+1}^{1}(0)_{i=1+1, \ldots, n}\right.
\end{array}\right.
$$

in the whole cone $K^{n}[0,1]$, respectively.

If now $l>1$ or $l>k$ and there exists a subset $I \in\{1, \ldots, l\}$ or $I \in\{k+1, \ldots, l\}$, respectively, such that for any $i \in I$ there is a $j=j_{i} \in I$ with $\Delta_{i j_{j}}>0$, then we have

$$
\left(B_{0} u_{0}\right)(t) \geq\left(\delta / 12 \lambda_{0}\right)\left(u_{0}\right)(t),
$$


where the components of $u_{0}$ are given by

$$
u_{o i}= \begin{cases}t(1-t) & \text { as } i \in I \\ 0 & \text { as } i \in I\end{cases}
$$

and $\delta=\min _{j \in I} \Delta_{i j}, \lambda_{0}=2 k_{0}, k_{0}=\max _{j \in I} k_{i}$.

Applying Theorem 5.7 of [9] to the problem (11), we obtain

Theorem 1: If $I>1$ in (10) and there exists a non-void subset $I \in\{1, \ldots, J\}$ such that for any $i \in I$ there is a $j=j_{j} \in I$ with $\Delta_{i j}>0$, beside $\Delta_{i j} \geq 0$ for all $i, j=1, \ldots, n$, then the equation (11) has a continuous branch with length 1 of eigenfunctions in the cone $K$ " $[0,1]$, i.e. the integral equation system (9) possesses a set of non-negative continuous solutions to all possible $a>0$ which has a non-void intersection with the boundary of any open set in $C^{n}[0,1]$ containing the zero point and is contained together with its closure in the open unit ball of $C^{n}[0,1]$.

If $l>k$ in (10) and there exists a non-void subset $I \in\{k+1, \ldots, I\}$ such that for any $i \in I$ there is a $j=j_{i} \in I$ with $\Delta_{i j}>0$, beside $\Delta_{i j} \geq 0$ for all $i, j=1, \ldots, n$, then the equation (11) has a continuous branch with length $\infty$ of eigenfunctions in the cone $K^{n}[0,1]$, i.e. the set of non-negative continuous eigenfunctions in the integral equation system (9) contains such ones with arbitraty large norm.

\section{Special cases}

In the special cases, where all $m_{i}>2$ or all $m_{j}$ satisfy $1<m_{i}<2$ or all $m_{i}=2(j=1, \ldots, n)$, respectively, more concrete existence assertions can be given for general kernels $K_{i j}$ analogously to the case of one equation (1). Namely, by the above assumptions the kernels $k_{i j}$ in (9) are non-negative continuous functions on $[0,1] \times[0,1] \times[0, \infty)$ satisfying the conditions $k_{i j}(0, \tau ; a)=0=k_{i j}(1, \tau ; a)$ and $k_{i j}(t, \tau ; 0)=0$. Therefore, for any $a>0$ the linear operators $B_{i j}$ defined by

$$
\left(B_{i j} y\right)(t)=\int_{0}^{t} k_{i j}(t, \tau ; a) y(\tau) d \tau
$$

are compact mappings in the Banach space $C[0,1]$ of continuous functions which leave the cone $K[0,1]$ of non-negative functions from $C[0,1]$ invariant. Moreover, if $\Delta_{i j}>0$, for any $y \in K[0,1], y \neq 0$, there exist positive numbers $\rho_{1}$ and $\rho_{2}$ such that the inequality

$$
p_{1} \cdot t(1-t) \leq\left(B_{i j} y\right)(t) \leq p_{2} \cdot t(1-t), t \in[0,1],
$$

holds (cf. [16]).

The integral equation system (9) has the form of the operator equation

$$
\Psi=A \Psi, A \Psi=B \Psi^{P}, \text { in } K^{n}[0,1]
$$

where $\Psi=\left(\Psi_{1}, \ldots, \Psi_{n}\right), \Psi^{P}=\left(\Psi_{2}^{P_{1}}, \ldots, \Psi_{n}^{P_{n}}\right)$ and the linear operator $B$ is defined by

$$
(B z)(t)=\left(\sum_{j=1}^{n}\left(B_{i j} z_{j}\right)(t)\right)_{i=1, \ldots . n}
$$

for $z=\left(z_{1}, \ldots, z_{n}\right)$. Clearly, for any $a>0$ the operator $A$ is a completely continuous mapping 
in $C^{n}[0,1]$ which leaves the cone $K^{n}[0,1]$ invariant and maps the zero element into itself.

As in [16] applying the Theorem 4.12 with Theorems 6.3 and 6.6 and the Theorem 4.14 of [9] to the equation (13), respectively (cf. [9: Chapt. 7, § 4.6]), we obtain

Theorem 2: If all $m_{i}>2(i=1, \ldots, n)$, or all $m_{i}$ satisfy $1<m_{j}<2(i=1, \ldots, n)$ and there exists a non-void subset $l \in\{1, \ldots, n\}$ such that $\Delta_{i i}>0$ for $i \in l$, beside $\Delta_{i j} \geq 0$ for all $i, j=$ $1, \ldots, n$, then for any $a>0$ the integral equation system (9) has a non-trivial non-negative continuous solution $\Psi$. If for $i \in I$ there is $\Delta_{i j}>0$ for each $j=1, \ldots, n$, the solution $\Psi$ has the property

$$
\gamma_{1} \cdot t(1-t) \leq \Psi_{i}(t) \leq \gamma_{2} \cdot t(1-t), i \in \mathbb{l},
$$

where $0<\gamma_{1} \leq \gamma_{2}<\infty$. In the first case with $\Delta_{i j}>0$ for all $i, j=1, \ldots, n$ this solution is uniquely determined in $K^{n}[0,1]$ and can be calculated by successive approximations starting from an arbitrary non-zero function $\Psi_{0} \in K^{n}[0,1]$.

If all $m_{i}=2(i=1, \ldots, n)$, i.e., $p_{i}=1(i=1, \ldots, n)$, the proof of the corresponding theorem in [16] can be taken over, where we use Theorem 2.5 of [9] with $u_{0}$ defined by (12) with a non-void subset $l=\left\{i_{1}, \ldots, i_{p}\right\}, 1 \leq p \leq n$, of $\{1, \ldots, n\}$ for obtaining the existence of a positive eigenvalue of the linear operator $A$ for any $a>0$ and the $u_{0}$-positivity of $A$ with $u_{0}$ defined by (12) with $I=\{1, \ldots:, n\}$ for getting the uniqueness and simplicity of this positive eigenvalue, respectively.

Theorem 3: If all $m_{i}=2(i=1, \ldots, n)$, and there exists a subset $l=\left\{i_{1}, \ldots, i_{p}\right\}, 1 \leq p \leq n$, of $\{1, \ldots ., n\}$ such that $\Delta_{i_{1} i_{2}}>0, \Delta_{i_{2} i_{3}}>0, \ldots, \Delta_{i_{p} i_{1}}>0$ and $\Delta_{i_{1} j}>0$ for each $j=1, \ldots, n$, beside $\Delta_{i j} \geq 0$ for all $i, j=1, \ldots, n$, then there is an a $>0$ such that the integral equation s. $y^{-}$ stem (9) has a non-negative continuous solution $\Psi$ with the property (14). If $\Delta_{i j}>0$ for all $i, j=1, \ldots, n$ to this a $>0$ the corresponding solution $\Psi$ is uniquely determined apart from an arbitrary positive constant numerical factor.

Corollary: In the last case the value of a is uniquely determined, too, if in (14) the partial kernels $K_{i j}^{(1)}$ are convex functions in $(-\infty, 0]$ and the kernels $K_{i j}^{(2)}$ are concave functions in $[0, \infty)$.

REMARK: The eigenvalues $\mu(a)$ of the operator $A$ fulfil the estimation

$\mu(a) \geq\left(a^{2} / 12\right)\left(\Delta_{i_{1} i_{2}} \Delta_{i_{2} i_{3}} \ldots \Delta_{i_{p} i_{1}} / \lambda_{i_{1}} \lambda_{i_{2}} \ldots \lambda_{j_{p}}\right)^{1 / p}$,

where $\lambda_{i}=2 k_{i}$ since $n_{i}=2$.

\section{Particular solutions}

For the equation (1) in the case of a piecewise constant kernel

$$
K(x)= \begin{cases}\alpha & \text { as } x<0 \\ \beta & \text { as } x>0\end{cases}
$$

with $\Delta=\alpha-\beta>0$ for $m \neq 2$ the solution $\Phi$ is given implicitely by 


$$
2\left(D_{\lambda} / a\right) x= \begin{cases}\int_{0}^{B \Phi(x)}\left(1-s^{\lambda}\right)^{-1 / 2} d s & \text { if } 0 \leq x \leq a / 2 \\ D_{\lambda}+\int_{B \Phi(x)}^{1}\left(1-s^{\lambda}\right)^{-1 / 2} d s & \text { if } a / 2 \leq x \leq a\end{cases}
$$

where $D_{\lambda}=\sqrt{\pi} \Gamma(1+1 / \lambda) / \Gamma(1 / 2+1 / \lambda), \lambda=m /(m-1)$, and the parameters $a$ and $B$ are connected by the relation

$$
a B^{1-\lambda / 2}=\sqrt{2} \lambda D_{\lambda} / \sqrt{\Delta} \text {. }
$$

The speed $c$ and the total population $I=\int_{0}^{a} \varphi(s) d s$ are given by the formulae $c=(\alpha+\beta) I / 2$ and $l=4 \lambda D_{\lambda} / a B \Delta$, respectively.

For $m=2$ we have the explicit solution

$$
\varphi(x)=\Phi(x)=b \sin \sqrt{\Delta / 2} x, 0 \leq x \leq \pi / \sqrt{\Delta / 2},
$$

which is determined up to an arbitrary positive constant factor $b=1 / B$. The corresponding speed $c$ and total population $I$ are $c=b \sqrt{2 / \Delta}(\alpha+\beta)$ and $I=2 b \sqrt{2 / \Delta}$, respectively.

We briefly describe the behaviour of the solution (16) as $m \rightarrow 2$, i.e. $\lambda \rightarrow 2$, where $D_{\lambda}$ $\rightarrow \pi / 2$. For $a=\pi / \sqrt{\Delta / 2}$ by (17) we have

$$
B=\left(\frac{\lambda D_{\lambda}}{\pi}\right)^{\frac{1}{1-\lambda / 2}}=\left(\frac{\lambda}{\sqrt{\pi}} \frac{\Gamma(1+1 / \lambda)}{\Gamma(1 / 2+1 / \lambda)}\right)^{\frac{1}{1-\lambda / 2}} \text {. }
$$

Introducing $r=1-\lambda / 2$, we obtain

$$
\begin{aligned}
B & \sim\left(1-r+\frac{1}{\sqrt{\pi}}\left[\Gamma^{\prime}(3 / 2)-\frac{\sqrt{\pi}}{2} \Gamma^{\prime}(1)\right] r\right)^{1 / r} \\
& =(1-r+[1-\ln 2] r)^{1 / r}=(1-\ln 2 \cdot r)^{1 / r} \text { as } r \rightarrow 0
\end{aligned}
$$

(cf. [7, p. 11]) so that $B \rightarrow 1 / 2$. Therefore, the solution (16) converges to the solution (18) with $b=2$ and $I=4 \sqrt{2 / \Delta}$.

Moreover, for given value $I>0$ the corresponding solution (16), that is the solution in the interval $\left[0, a_{\lambda}\right]$ with $a_{\lambda}=I^{2 / \lambda-1} \Delta^{1 / \lambda-1} 2^{2-3 / \lambda} \lambda D_{\lambda}$, tends to the solution (18) with the same value of $I$, i.e. with $b=(I / 2) \sqrt{\Delta / 2}$.

\section{Numerical results}

In the last section we discuss the numerical approximaton of travelling waves for the representative special case of piecewise constant kernels (15) in equation (1) and indicate the dependence of the wave on the parameter $m$. For $m \neq 2$, because of (16), we only need to evaluate the function

$$
F(y)=\int_{0}^{y}\left(1-s^{\lambda}\right)^{-1 / 2} d s \text { for } y \in[0,1] .
$$

An approximation of $F^{-1}$ is not necessary, as the knowledge of

$$
\left(F\left(y_{i}\right) /\left(2 D_{\lambda} / a\right),\left(y_{i} / B\right)^{1 /(m-1)}\right)
$$

for sufficiently many $y_{i} \in[0,1]$ is sufficient to get a parametric representation of $z=\Phi(x)$, $x=[0, a / 2]$ (e.g. by linear splines). For the approximation of $F(y)$ for $y \in[0,1]$ we use the 
representation

$$
F(y)=1 / \lambda \int_{0}^{y} t^{1 / \lambda-1}(1-t)^{-1 / 2} d t=1 / \lambda B\left(y^{\lambda} ; 1 / \lambda, 1 / 2\right),
$$

where $B$ is the incomplete Beta Function. We have a weak singularity at $t=0$ and at $t=1$, if $y=1$. Therefore we write

$$
F(y)=1 / \lambda \int_{0}^{y^{\lambda}} t^{1 / \lambda-1}\left(y^{\lambda}-t\right)^{-1 / 2}\left(\frac{y^{\lambda}-t}{1-t}\right)^{1 / 2} d t
$$

and arrive at an integral with specific weak singularities at the lower and upper limit of integration. The remaining integrand is continuous. We approximate (19) by Gauss-Jacobi integration for these weight functions. The used weights and abscissae have been computed by the routine DOIBCF from the NAG FORTRAN LIBRARY, MARK 12 . For $m=2$ we use directly (18).

For all computations we take $\alpha=2, \beta=-1,200$ points for $y, 50$ points of integration.

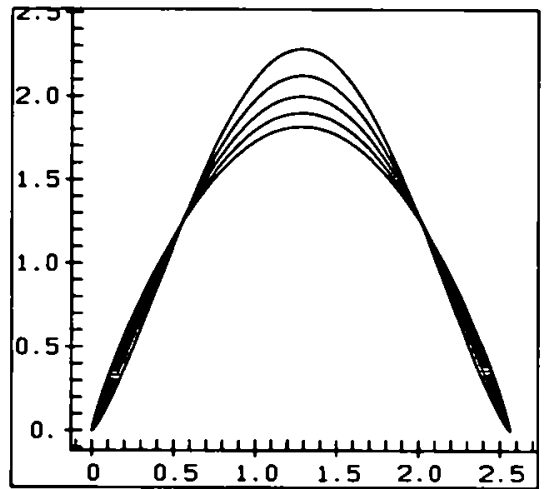

Fig.1 Travelling waves for fixed a: $m$ close to 2

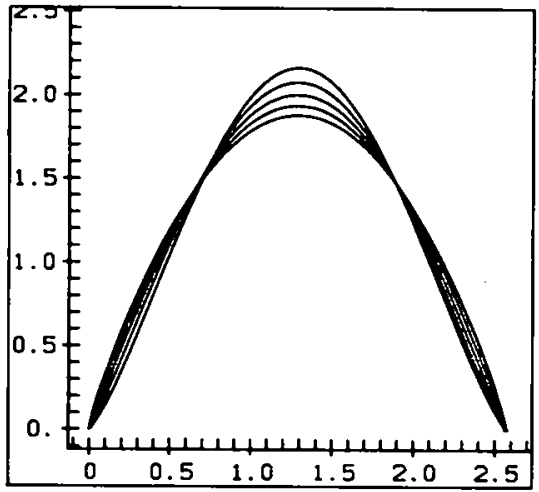

Fig. 2 Travelling waves for fixed $I: m$ close to 2

In Figure 1 we fix a to $a=\pi / \sqrt{\Delta / 2}$, the interval for which travelling waves exist for $m=2$. The displayed curves correspond to (with decreasing maximum): $m=1.8,1.9,2.0$, $2.1,2.2$, where for $m=2$ we have $b=2$ as predicted above. The convergence for $m \rightarrow 2$ is clearly to be seen, the total population $I$ (and hence the speed $c=(\alpha+\beta) / 2 \cdot I$ ) increase with decreasing $m$.

In Figure 2 we fix $I$ to $I=4 \sqrt{2 / \Delta}$, corresponding to $m=2$ for $a=\pi / \sqrt{\Delta / 2}$ from Figure 1. As above we show the curves for (with decreasing maximum): $m=1.8,1.9,2.0,2.1$, 2.2. Again the convergence for $m \rightarrow 2$ can be observed. The length of support a varies only slightly, decreasing for $m \downarrow 2$ and for $m \uparrow 2$, as can be seen from Table 1 .

\section{\begin{tabular}{|c|c|c|c|c|c|}
\hline an & 2.2 & 2.1 & 2.0 & 1.9 & 1.8 \\
\hline
\end{tabular}}

Table 1: The value of a for fixed $I$ (corresponding to Figure 2)

Finally we illustrate, for fixed $I$, namely $I=1$, the behaviour as $m \rightarrow \infty$ and $m \rightarrow 1$. In Figure 3 the waves corresponding to (with decreasing $a$ ): $m=2.5,5,10,100,1000$ are 
shown. The length of support a decreases to 1 and the waves approximate a rectangular shape. For the porous medium equation this phenomenon is connected with the notion "mesa problem". In Figure 4 we display the waves corresponding to (with increasing a) $m=1.9,1.8,1.7,1.6$.

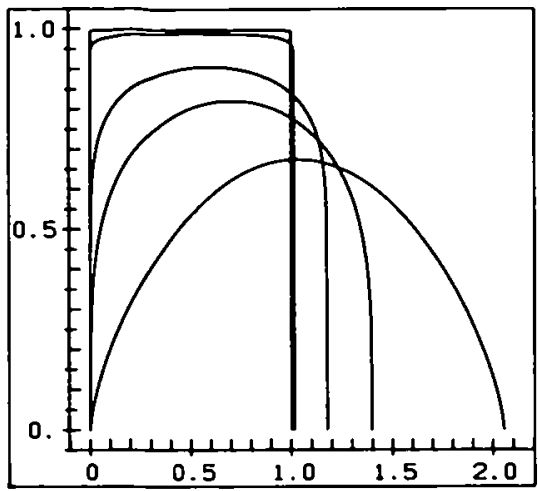

Fig. 3 Travelling waves for increasing $m$

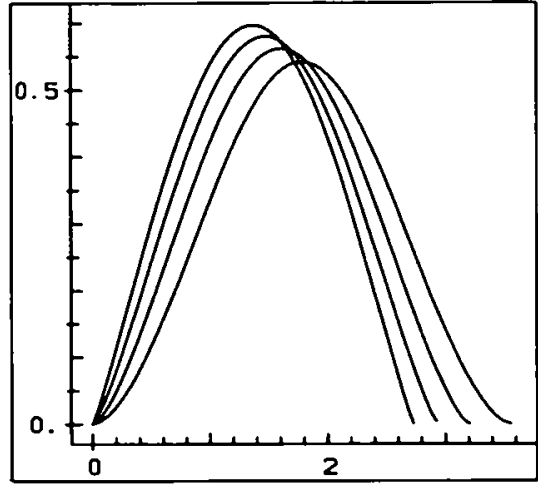

Fig. 4 Travelling waves for decreasing $m$

\section{REFERENCES}

[1] ALT, W.: Degenerate diffusion equations with drift functionals modelling aggregation. Nonlinear Analysis 9 (1985), 811 - 830.

[2] GREENBERG, J. M., and W. ALT: Stability results for a diffusion equation with functional drift approximating a chemotaxis model. Trans. Amer. Math. Soc. 300 (1987), $235-258$.

[3] GURNEY, W. S. C., and R. M. NISBET: The regulation of inhomogeneous populations. J. Theor. Biol. 52 (1975), $441-457$.

[4] GURTiN, M. E. , and R. C. MAC CAMY: On the diffusion of biological populations. Math. Biosci. 33 (1977), 35 - 49.

[5] IKEDA, T.: Standing pulse-like solutions of a spatially aggregating population model. Japan. J. Appl. Math. 2 (1985), 111 - 149.

[6] IKEDA, T.. and T. NAGAI : Stability of localized stationary solutions. Japan. J. Appl. Math. 4 (1987), $73-97$.

[7] JAHNKE-EMDE-LÖSCH: Tafeln Höherer Funktionen. Stuttgart: B. G. Toubner Verlagsges. 1966.

[8] KNABNER, P., and L. V. WOLFERSDORF: Travelling wave solutions of some systems of nonlinear diffusion equations with integral term. Anwendungsbezogene Optimierung und Steuerung. Report 123 (1989), 1 - 22.

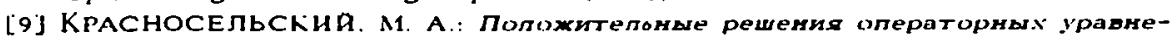
Mstr. Mlocxea: Onзмaтrus 1962. - Engl. transl.: KRASNOSELSKII, M. A.: Positive Solutions of Operator Equations. Groningen: Noordhoff 1964.

[10] MIMURA. M., and J. SATSUMA: Ecotons of a nonlinear diffusion equation. In: Development of Soliton Theory. Kyoto: University, Res. Inst. Math. Sci. 1985, $184-199$.

[11] MIMURA, M., and M.YAMAGUTI : Pattern formation in interacting and diffusing systems in population biology. Adv. in Biophys. 15 (1982), $19-65$.

[12] NAGAI. T.: Some nonlinear degenerate diffusion equations with a nonlocally convective term in ecology. Hiroshima Math. J. 13 (1983), 165 - 202.

[13] NAGAI, T., and M. MIMURA: Some nonlinear degenerate diffusion equations related 
to population dynamics. J. Math. Soc. Japan 35 (1983), 539 - 562.

[14] NAGAI, T., and M. MIMURA: Asymptotic behavior for a nonlinear degenerate diffusion equation in population dynamics. SIAM J. Appl. Math. 43 (1983), $449-464$.

[15] NACAI, T., and M. MIMURA: Asymptotic behaviour of the interfaces to a nonlinear degenerate diffusion equation in population dynamics. Japan. J. Appl. Math. 3 (1986). $129-161$.

[16] WOLFERSDORF.L. V.: Travelling wave solutions of a nonlinear diffusion equation with integral term. Z. Anal. Anw. 9 (1990), 303 - 312.

Received 14.11. 1989

Dr. Peter Knabner

Mathematisches Institut

der Universität Augsburg

Universitatsstr. 6

$D($ West $)$ - 8900 Augsburg
Prof. Dr. Lothar von Wolfersdorf

Fachbereich Mathematik

der Bergakademie Freiberg

Bernhard - von - Cotta - Str. 2

$\mathrm{D}(\mathrm{Ost})$ - 9200 Freiberg

\section{Book review}

A. Friedman: Mathematics in Industrial Problems, Part 3 (The IMA Volumes in Mathematics and its Applications: Vol. 31). Berlin - Heidelberg - New York: Springer - Verlag 1990, 187 pp., 96111.

As the title shows, this is already the third volume in the IMA series which is devoted to the applications of mathematics in industrial problems. It bases on presentations in the seminar on Industrial Problems at IMA from October 1989 to May 1990. The contributions are ordered chronically and cover a wide range of applications. Each of them is self-contained.

The book consists of 18 chapters with almost the same structure: The problem is described, then the mathematical model follows and the solution knoun so far. Finally there is a list of open problems. References are given for each chapter separately. The last chapter shows that work on open problems goes on, it contains solutions (or references to solutions) to some of the open problems from Part 2.

Most of the problems belong to mathematical analysis. They arise from physical, chemical and technological models. Only a minor part of the book deals with problems from other mathematical disciplines as probability theory, statistics or optimization.

Contents: 1. Internal oxidation of binary alloys; 2 . Fundamental problems in the theory of shaped charged jets; 3 . Mathematical modeling of dielectric waveguides; 4 . A diffusion problem from rock porosity measurements; 5 . Applications and modeling of diffractive optical elements; 6 . An approach to optimal classification; 7. Polymer-dispersed liquid crystal films for light control; 8. Singularity problems in the stress analysis of semiconductor packging; 9. Pulse reflection from a randomly stratified medium; 10. Theory of polymer melt viscoelasticity; 11 . The advection equation in air quality modeling; 12. Diffusion in swelling media: modeling and applications; 13 . Mathematical modeling of semiconductor lasers; 14 . Conformation of random polymers; 15 . Current-voltage relations for electrolytic solutions; 16 . Scaling and optimization for list-matching; 17. Topics in tomography. 